Original Article - Clinical Science

\title{
Minimally invasive glaucoma surgery: comparison of iStent with iStent inject in primary open angle glaucoma
}

\author{
Joobin Hooshmand MBBS, ${ }^{1}$ Philip Rothschild, ${ }^{1,2}$ Penny Allen $\mathrm{PhD},{ }^{1,2}$ Nathan M Kerr \\ MD FRANZCO, ${ }^{3}$ Brendan J Vote FRANZCO ${ }^{1}$ and Tze'Yo Toh FRANZCO ${ }^{1}$
}

1. Tasmanian Eye Institute, Launceston, Tasmania, Australia

2. Clinical School, University of Tasmania, Launceston, Tasmania, Australia

3. Royal Victorian Eye and Ear Hospital, Melbourne, Victoria, Australia

Correspondence: Dr Joobin Hooshmand, Launceston Eye Institute, 36 Thistle St

West, Launceston, Australia 7250

Email: joobin.hooshmand@gmail.com

Short running title: iStent comparison with iStent inject

Received 7 August 2018; accepted 23 April 2019

Funding sources / Financial disclosure: None

Conflict of interest: None

This is the author manuscript accepted for publication and has undergone full peer review but has not been through the copyediting, typesetting, pagination and proofreading process, which may lead to differences between this version and the Version of Record. Please cite this article as doi: $10.1111 /$ ceo.13526

This article is protected by copyright. All rights reserved. 


\section{ABSTRACT}

Importance: Minimally Invasive Glaucoma Surgery has gained significant traction in recent years. This study evaluates the first and second-generation trabecular micro-bypass stents 'iStent' and 'iStent inject'.

Background: To evaluate and compare the effect of a single iStent and double iStent inject in Primary Open Angle Glaucoma.

Design: Prospective comparative interventional case series.

Participants: Primary Open Angle Glaucoma patients undergoing trabecular microbypass stent insertion combined with cataract surgery.

Methods: Baseline demographic information, pre-operative, intra-operative and post-operative outcomes including intraocular pressure (IOP), visual acuity, reliance on glaucoma medication and complications were collected and analysed.

Main Outcome Measures: Primary, secondary and tertiary outcome measures were consecutively defined as an IOP of $\leq 18 \mathrm{mmHg}$ with zero medications, an IOP of $\leq 18 \mathrm{mmHg}$ with reduced medications or a $20 \%$ reduction in IOP with or without medication.

Results: The study comprised 145 eyes in the iStent and 100 eyes in the iStent inject group. At 12 months, $56.0 \%$ of the iStent and $51.3 \%$ of the istent inject eyes had achieved primary success and $63.1 \%$ and $57.7 \%$ secondary success. The mean post-operative IOP was $16.6 \mathrm{mmHg}$ in iStent and $16.9 \mathrm{mmHg}$ in iStent inject. Survival analysis demonstrated a greater incidence of failure in the iStent inject beyond 5 months.

Conclusions: Both trabecular micro-bypass stents in this study were effective in reducing IOP and the burden of medication when combined with cataract surgery. There was no statistically significant difference between the two groups across our outcome measures although the iStent inject required earlier recommencement of medications for optimal IOP control. 
Keywords: Minimally Invasive Glaucoma Surgery, MIGS, iStent, iStent inject, cataract surgery

\section{INTRODUCTION}

Minimally Invasive Glaucoma Surgery (MIGS) has gained significant traction over the past few years. New devices and procedures are constantly being developed in an effort to lower intraocular pressure (IOP) in a less invasive and purportedly safe manner. In Australia, there has been a marked increase in MIGS uptake with Medicare data indicating an exponential increase in use over the last few years. ${ }^{1,2}$ Although MIGS is currently seen primarily as adjunct to cataract surgery, it is increasingly being considered as a viable initial treatment option. ${ }^{3}$ The objective of this study was to evaluate and compare the first and secondgeneration trabecular micro-bypass stents, 'iStent' and 'iStent inject' (Glaukos Corporation, CA, USA). Trabecular stent bypass microsurgery aims to reduce intraocular pressure by creating a bypass channel between the anterior chamber and Schlemm's canal to improve the drainage of aqueous humour. iStent was the first ab-interno micro-bypass device of its kind based on this principal. The $1 \mathrm{~mm}$ long, Lshaped titanium device is implanted via a preloaded injector through the trabecular meshwork under gonioscopic guidance. Once inside the trabecular meshwork, a well placed stent provides aqueous outflow directly into collector channels.

Figure 1: A. First generation trabecular micro-bypass stent (iStent). B. Second generation trabecular micro-bypass stent, (iStent inject). Image supplied.

Although successful in reducing the IOP, the iStent was superseded by iStent inject, a much smaller second generation trabecular micro-bypass stent. The iStent inject is 
a $0.4 \mathrm{~mm}$ long, $0.3 \mathrm{~mm}$ wide plug shaped implant with a central opening that is injected into the trabecular meshwork under gonioscopic guidance. The stent was redesigned for easier insertion and its injector is pre-loaded with two stents inserted approximately 2 to 3 clock hours away from each other. (Figure 1 )

A number of studies have demonstrated the effectiveness of iStent and iStent inject. In a systematic review and meta-analysis of 2,143 patients from 32 studies, comparing stent insertion combined with phacoemulsification against phacoemulsification alone in patients with glaucoma and cataract, there was a statistically significant decrease in IOP from baseline in the combined group compared with the phacoemulsification only group at a follow up of 12- 58 months. ${ }^{4}$ The meta-analysis also demonstrated a significant reduction in the number of glaucoma medications used after the procedure in the combined group compared with the phacoemulsification only group (weighted mean reduction of 1.33 from baseline compared with 1.01). ${ }^{4}$ Significant reduction in IOP from baseline has also been demonstrated in patients with mild to moderate glaucoma treated with stent insertion alone at follow up of 6-18 months. ${ }^{5}$

Although each trabecular micro-bypass device has proven to be efficacious, the two devices have not been previously compared in performance and efficacy.

\section{METHODS}

\subsection{Study design}

We conducted a prospective comparative interventional case series on patients with mild to moderate primary open angle glaucoma (POAG) and cataracts. Patients undergoing cataract surgery combined with stent insertion were consecutively recruited from two centres across Launceston, Tasmania, Australia. Mild to moderate POAG was defined as having a vertical cup to disc ration of 0.8 or less with stable serial IOPs and visual field assessments. All patients were seen and operated on by 
a single senior glaucoma specialist with extensive experience in trabecular stent micro-bypass surgery. iStent was considered well placed when only the "snorkel" component was visible with the rest of the implant inside the Schlemm's canal. iStent inject was considered well placed when the level of the "collar" was flush with the trabecular meshwork. The study was conducted in accordance with the tenets of the Declaration of Helsinki; ethics approval was obtained from the Tasmanian Human Research Ethics Committee (H0014226).

Baseline demographic information as well as data on IOP, visual acuity and reliance on the number of glaucoma medications were recorded pre and post operatively. Intraoperative observations on device performance and complications were made. Patients were seen at day 1 and week 3-4 post operative at which point all glaucoma medications were ceased. The post-operative steroid regime was kept identical between the two groups. Follow up occurred every 3 months or as otherwise clinically indicated. Patients were recommenced on monotherapy if IOP was greater than $18 \mathrm{mmHg}$ in any follow up visit; an IOP of $18 \mathrm{mmHg}$ was chosen as it has been shown to reduce the long term progression of visual field defects. ${ }^{6}$ Further agents were added one at a time at each follow up if clinically indicated. A cut off of 18 months was chosen as very few patients had passed this milestone in the second generation device, iStent inject, group.

\subsection{Outcome measures}

- Primary success was defined as an IOP of $18 \mathrm{mmHg}$ or less with zero number of topical glaucoma medications.

- Secondary outcome was defined as an IOP of $18 \mathrm{mmHg}$ or less with reduced number of topical glaucoma medications.

- Tertiary outcome was defined as a $20 \%$ reduction in IOP with or without topical glaucoma therapy. 


\subsection{Statistical analysis}

Patient data entered into Excel (Microsoft Inc, WA, USA) were extracted and imported into Stata 15 (StataCorp LLC, TX, USA) for analysis. Numbers of eyes and percentages are reported for categorical data and means with standard deviations for continuous data. Cross-tabulations were produced to compare categorical data in the iStent and iStent inject groups, with chi-square tests used to evaluate statistically significant differences. Independent t-tests were used to investigate mean IOP, number of glaucoma medications and visual acuity (logMAR) comparison in the iStent and iStent inject groups. A Kaplan-Meier survival curve plotted time to glaucoma medication re-initiation after surgery within 18 months of follow-up and median time to drop re-initiation was calculated.

\section{RESULTS}

The study comprised 245 eyes from 148 patients, aged 53-89 years at the time of the operation (Mean 71, SD 7.1). There were 145 eyes in the iStent and 100 eyes in iStent inject groups. All patients had a confirmed diagnosis of POAG. The mean preoperative IOP was identical in both groups $(18.9 \mathrm{mmHg})$. The mean pre-operative number of topical agents was 1.7 in the iStent and 1.6 in the iStent inject group. Patient demographics and baseline characteristics are shown in table 1.

Table 1: Patient demographics and baseline characteristics

\begin{tabular}{lcccc}
\hline \multicolumn{5}{c}{ Mean (SD) or $\mathrm{n}(\%)$} \\
\hline Variable & Both & iStent & $\begin{array}{c}\text { iStent } \\
\text { inject }\end{array}$ & $\begin{array}{c}\boldsymbol{P} \\
\text { value }\end{array}$ \\
\hline Eyes (n) & 245 & 145 & 100 & - \\
Right (n) & 120 & $73(50.3)$ & $47(47.0)$ & 0.61 \\
Age (years) & $(49.0)$ & & & \\
& $71(7.1)$ & 70.2 & 70.9 & 0.40
\end{tabular}


Gender (female)

Pre-operative IOP $(\mathrm{mmHg})$

Pre-operative agents (n)

Pre-operative visual acuity (logMAR)

(Snellen equivalent)
135

(55.3)

$18.9(5.2)$

$1.6(0.1)$

$0.2(0.1)$

$87(60.4)$

$48(48.0)$

0.06

18.9

(4.7)

$18.9(5.9)$

0.93

.7 (0.1)

$1.6(0.1)$

0.64

$0.2(0.1)$

$0.1(0.1)$

0.28

9.5

7.5

\subsection{Outcome measures}

At 12 months, $56.0 \%$ of the iStent and $51.3 \%$ of the iStent inject eyes had achieved primary success and $63.1 \%$ and $57.7 \%$ secondary success. Tertiary success was achieved in $34.8 \%$ in the iStent and $29.5 \%$ in the iStent inject eyes. Forty-three eyes reached 18 months of follow up in the iStent inject group due to later introduction of the device. The main outcome measures achieved at different time points across the study are outlined in table 2 .

Table 2: Primary, secondary and tertiary outcome comparison

\begin{tabular}{ccccc}
\hline Time (Months) & Both & iStent & iStent inject & P value \\
\hline & \multicolumn{4}{c}{ Primary Success $\mathrm{n}(\%)$} \\
3 & $158(67.8)$ & $93(67.4)$ & $65(68.4)$ & 0.87 \\
6 & $148(63.2)$ & $94(65.3)$ & $54(60.0)$ & 0.42 \\
12 & $119(54.3)$ & $79(56.0)$ & $40(51.3)$ & 0.50 \\
18 & $84(47.5)$ & $65(48.5)$ & $19(44.2)$ & 0.62 \\
\hline & \multicolumn{5}{c}{ Secondary Success $\mathrm{n}(\%)$} \\
6 & $164(70.4)$ & $98(71.0)$ & $66(69.5)$ & 0.80 \\
12 & $161(68.8)$ & $105(72.9)$ & $56(62.2)$ & 0.09 \\
18 & $134(61.2)$ & $89(63.1)$ & $45(57.7)$ & 0.43 \\
3 & $102(57.6)$ & $81(60.4)$ & $21(48.8)$ & 0.18 \\
6 & & \multicolumn{2}{c}{ Tertiary Success $\mathrm{n}(\%)$} & \\
12 & $68(37.8)$ & $51(37.0)$ & $37(38.9)$ & 0.76 \\
18 & $72(28.6)$ & $42(29.2)$ & $25(27.8)$ & 0.82 \\
& $67(37.9)$ & $49(34.8)$ & $23(29.5)$ & 0.43 \\
& & $51(38.1)$ & $16(37.2)$ & 0.92 \\
\hline
\end{tabular}


$\mathrm{N}=$ number; $\%=$ percentage; Total number of eyes for both groups $\mathrm{n}=233$ at 3 months, 234 at 6 months, 219 at 12 months and 177 at 18 months. There were 43 eyes that reached 18 months follow up in the iStent inject group.

\subsection{Intraocular pressure}

The mean IOP was generally reduced over the study period however an uptrend was noted in the iStent inject eyes from 6 months following surgery. Figure 2 depicts the mean IOP for the iStent and iStent inject groups pre-operatively and at each followup timepoint. For each timepoint, there was no significant difference in mean IOP between the groups. Raw IOP data pre-operatively compared with 12 months postoperative are shown in Figure 3.

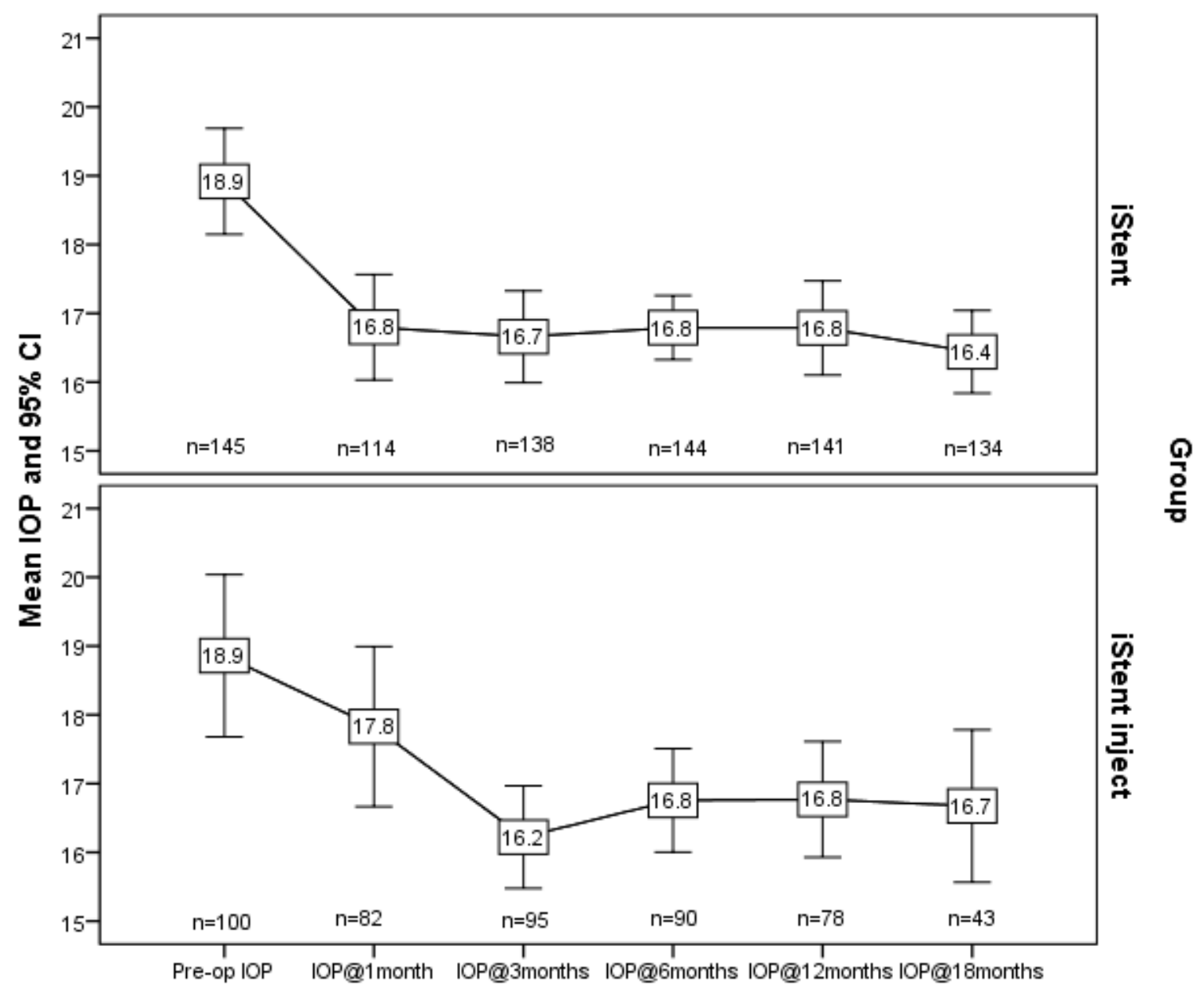

This article is protected by copyright. All rights reserved. 
Figure 2: Error bar graphs demonstrating the mean IOP trend in iStent (top) and iStent inject (bottom).

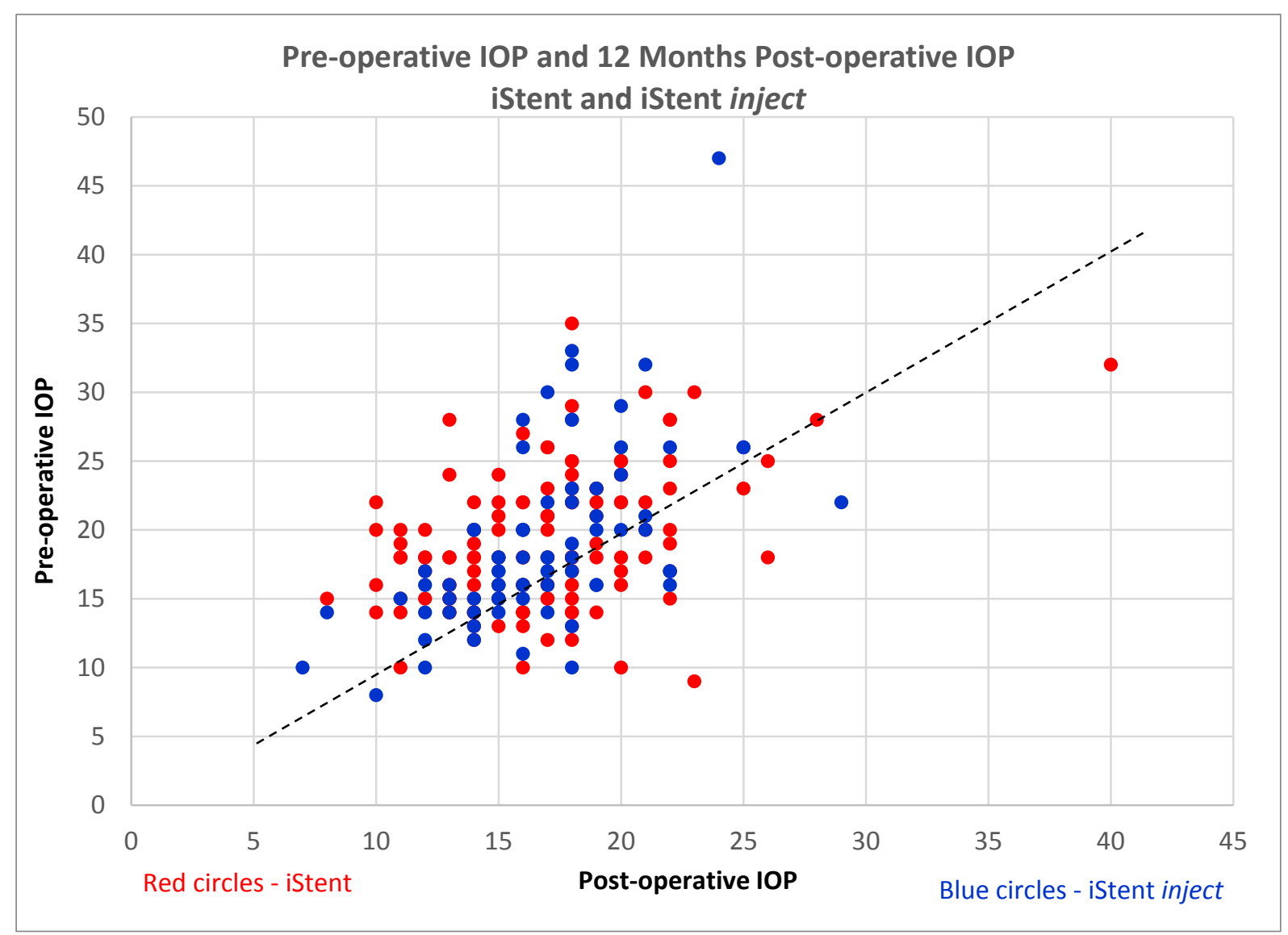

Figure 3: Scatter plot graph of the raw IOP data pre-operatively compared with 12 months post-operative for iStent and iStent inject eyes. Data points above the line indicate a reduction in IOP, those on the line indicate no change with those below the line indicating an increase in post-operative IOP.

\subsection{Glaucoma medication}

The mean number of glaucoma medications used reduced from 1.7 to 0.6 in the iStent group and 1.6 to 0.7 in iStent inject eyes. An overview of the reduction in the mean number of the glaucoma medications used is demonstrated in Figure 4. The 
number of eyes and the distribution of the number of glaucoma agents required in the pre-operative compared with the post-operative period is demonstrated in Figure 5.

By 12 months, 90 of the $141(63.8 \%)$ iStent and 52 of the $79(66.7 \%)$ iStent inject eyes had recommenced topical glaucoma therapy. Of those who required recommencement of topical therapy, the mean time to drop initiation was 12 months for iStent and 7 months for iStent inject. Overall, $49 \%$ of the eyes did not require topical therapy during the follow up period.

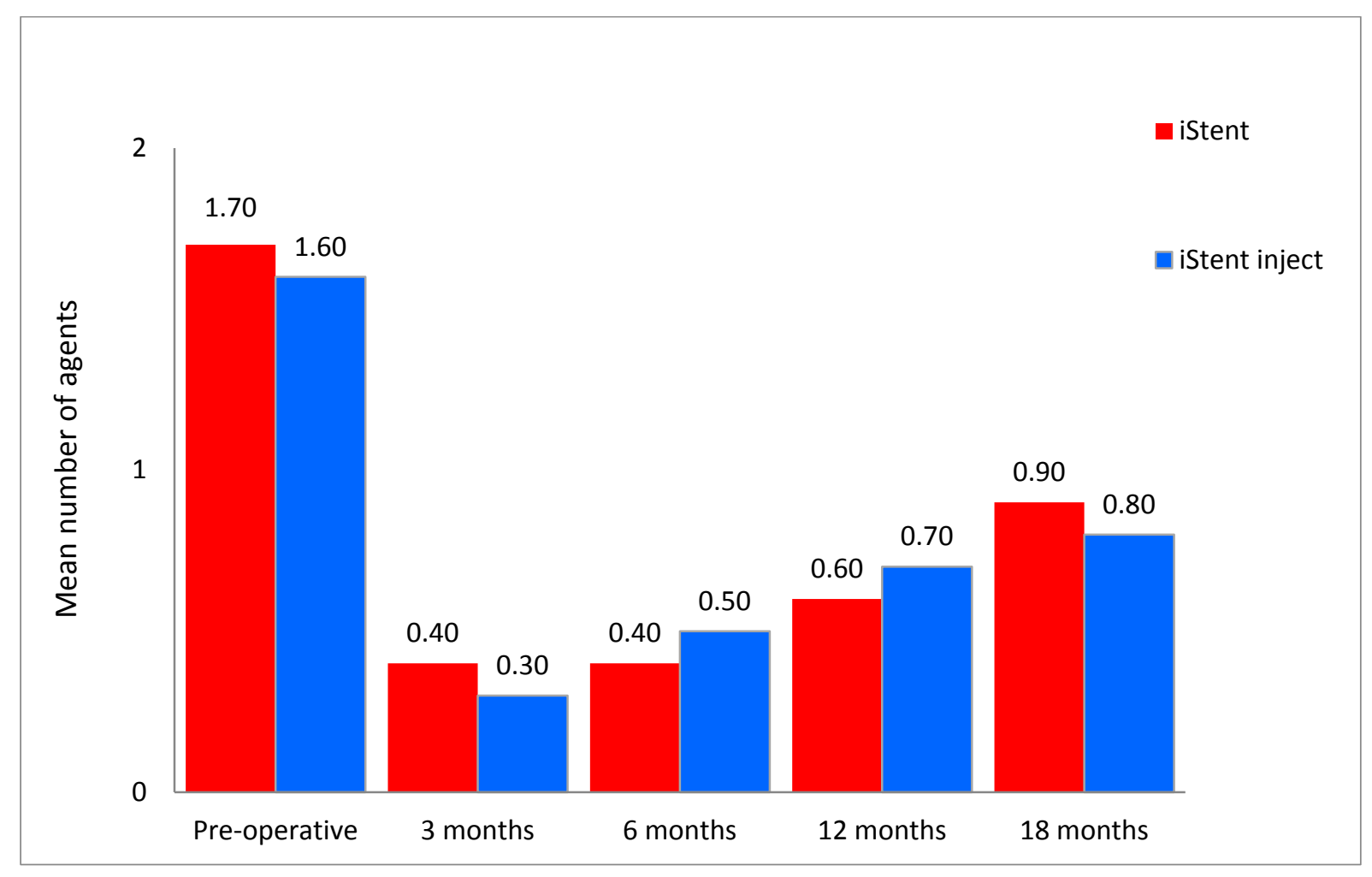

Figure 4: Mean number of glaucoma agents used over 18 months. 


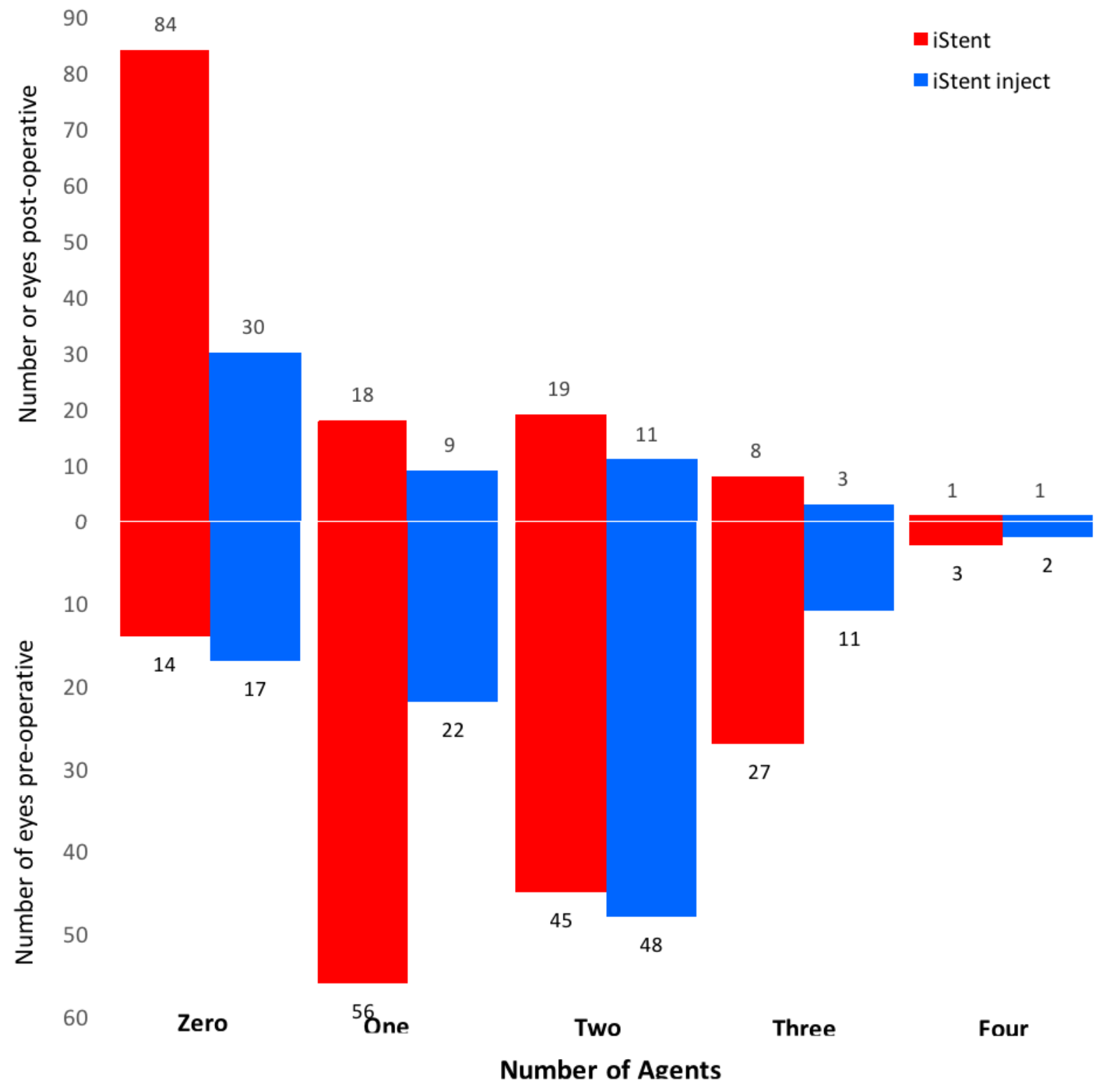

Figure 5: Number of eyes ( $y$ axis) and the distribution of the number of glaucoma agents ( $x$ axis) required in the pre-operative (bottom) compared with the postoperative (top) period. 
A very similar survival experience (estimated time to topical therapy initiation post surgery) was demonstrated in both groups for up to 5 months post-operative utilising the Kaplan - Meier survival estimator. From 5 months on, the survival curves begin to diverge, with the greater incidence of failure (defined as the initiation of eye drops) in the iStent inject eyes (Figure 6).

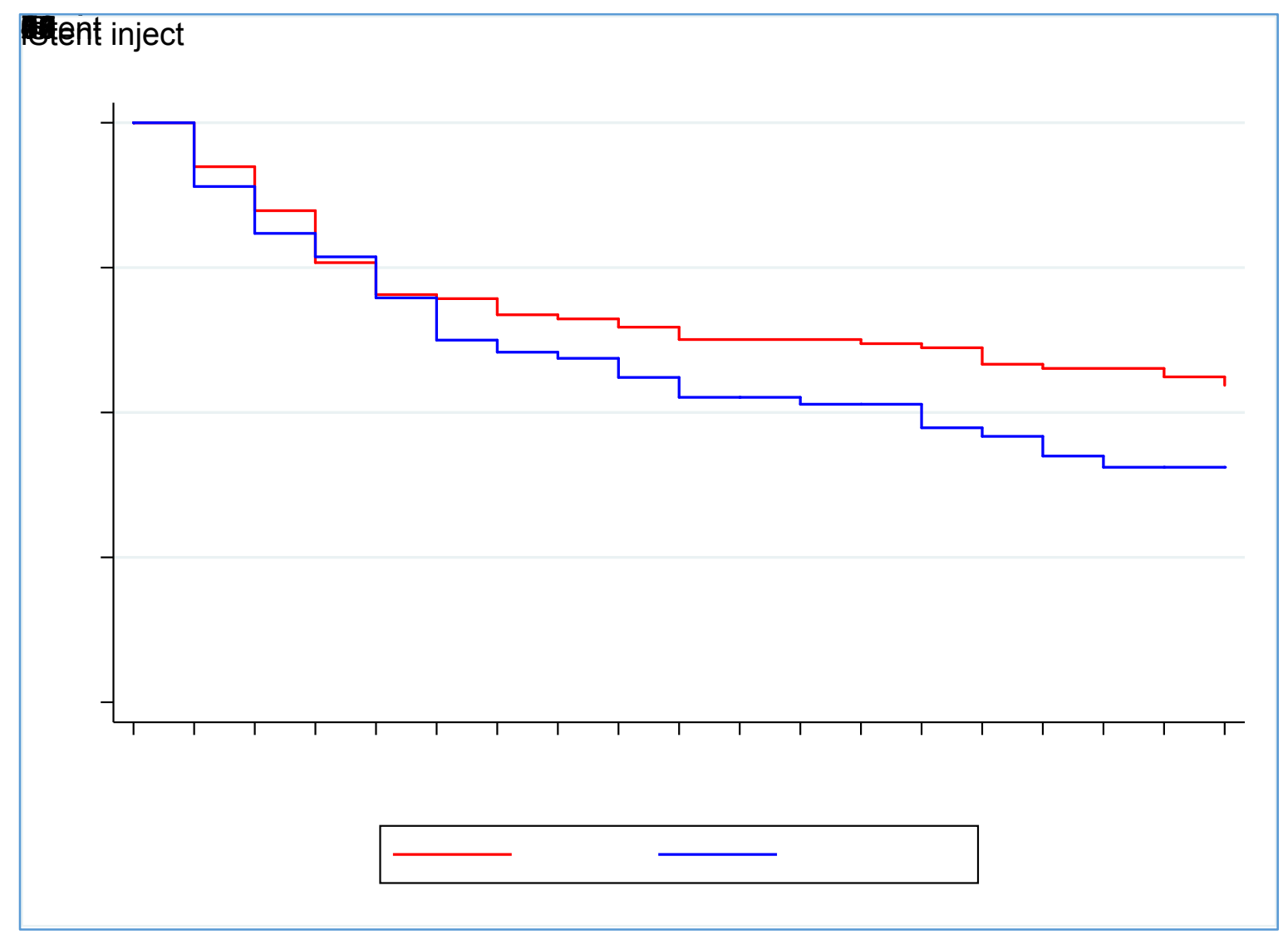

Figure 6: Kaplan-Meier survival analysis demonstrating time to post-operative initiation of glaucoma medications.

\subsection{Visual acuity}

The mean best corrected visual acuity at 12 months was 0.09 logMAR (SD 0.11 ) or $6 / 7.5$ Snellen equivalent, with no significant difference between the two groups ( $t$ $(188)=-0.8, p=0.41)$. 


\subsection{Complications}

Hyphaema was the most common complication observed with higher incidence in the iStent eyes. These were conservatively managed with no further complications. Corneal abrasions, corneal oedema, implant failure and stent malposition made up the remainder of the small number of complications observed (Table 3.) Only 1 patient implanted with iStent inject, presented with a delayed spontaneous nontraumatic macro-hyphaema 4 months post implant insertion. This was conservatively managed and resolved without further complications.

Table 3: Complications

\begin{tabular}{|c|c|c|c|c|}
\hline \multirow{6}{*}{ 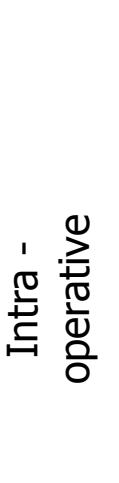 } & Complication & $\begin{array}{c}\text { iStent [n = } \\
145]\end{array}$ & $\begin{array}{c}\text { iStent inject [n = } \\
100]\end{array}$ & $\begin{array}{c}P \\
\text { value }\end{array}$ \\
\hline & Bleeding in $\mathrm{AC}$ & $13(8.9 \%)$ & $4(4.0 \%)$ & 0.13 \\
\hline & Corneal abrasion & $3(2.1 \%)$ & 0 & 0.27 \\
\hline & Failure to implant & $1(0.7 \%)$ & 0 & 1.00 \\
\hline & $\begin{array}{l}\text { Failure to implant } 2 \\
\text { stents }\end{array}$ & $\mathrm{N} / \mathrm{A}$ & $2(2.0 \%)$ & $\mathrm{N} / \mathrm{A}$ \\
\hline & Stent malposition & $3(2.1 \%)$ & $1(1.0 \%)$ & 0.65 \\
\hline \multirow{4}{*}{ 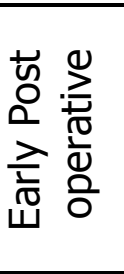 } & Hyphaema & $14(9.7 \%)$ & $6(6.0 \%)$ & 0.35 \\
\hline & Corneal oedema & $3(2.1 \%)$ & $1(1.0 \%)$ & 0.65 \\
\hline & Corneal abrasion & $1(0.7 \%)$ & $1(1.0 \%)$ & 1.00 \\
\hline & \multicolumn{4}{|c|}{$\mathrm{AC}=$ anterior chamber $; \mathrm{NA}=$ not applicable } \\
\hline
\end{tabular}

\section{DISCUSSION}

The first and the second generation trabecular micro-bypass stents are both proven to be efficacious in reducing IOP and reliance on glaucoma medications in mild to moderate glaucoma. Despite the relative ease in intraoperative insertion of these 
implants under gonioscopic guidance, these ab-interno micro-stents are subject to intraoperative malpositionning which can result in failure to implantation or luminal obstruction and decreased efficacy. ${ }^{7,8}$ The $1 \mathrm{~mm}$ long, $L$ shaped first generation device, iStent, is slightly more difficult to implant owing to its size and shape. Higher incidence of intraoperative hyphaema is also reported with occasional recurrence and lumen blockage requiring implant removal. ${ }^{9-11}$ The second generation device, iStent inject, is a much smaller implant at $0.4 \mathrm{~mm}$ long and was specifically designed for ease of insertion. The iStent inject's pre-loaded injector is also equipped with 2 implants usually utilised at the same time, thereby reducing the impact of single implant blockage.

Our results confirm the efficacy of each stent consistent with other published literature to date. In a randomised controlled clinical evaluation of the trabecular micro-bypass stent (iStent) with phacoemulsification in patients with glaucoma and cataract $72 \%$ achieved primary outcome success. ${ }^{12}$ The nominated IOP to recommence treatment in this study was however set higher at $21 \mathrm{mmHg}$. We elected for a more conservative approach and chose to recommence treatment at $18 \mathrm{mmHg}$ based on evidence from the Advanced Glaucoma Intervention Study (AGIS). ${ }^{6}$ Interestingly $50 \%$ of the control eyes in the randomised controlled trial achieved an IOP of less than $21 \mathrm{mmHg}$ following cataract surgery alone. ${ }^{12}$ Our study found $56.0 \%$ primary success at 12 months in the iStent group and $51.3 \%$ in the iStent inject group. Given the small difference between the groups, a post-hoc power calculation found more than 1500 patients would need to be recruited to each group to detect a significant difference at $80 \%$ power and $a=$ 0.05 .

We compared the effects of both stents in a prospective study of POAG patients with very similar if not identical demographics and baseline characteristics. Although there was no statistically significant difference between the two groups across our outcome measures we noted iStent inject patients require earlier recommencement 
of topical glaucoma therapy for optimal IOP control. We postulate this to be due to the dynamics of fluid flow through the stent lumen with the iStent having a larger drainage pathway than that of the iStent inject. The higher incidence of complications associated with iStent observed in our cohort are statistically not significant and bore no bearing on our patient's final visual outcomes.

\subsection{Conclusion}

Both trabecular micro-bypass stents in this study reduced IOP by a mean of $2.0-$ $2.3 \mathrm{mmHg}$ and reduced the number of medications by $0.8-1.0$. There was no statistically significant difference between the two groups across our outcome measure. Both devices appear to be safe with no significant visual or IOP related complications. 


\section{REFERENCES}

1. Kerr NM, Kumar HK, Crowston JG, Walland MJ. Glaucoma laser and surgical procedure rates in Australia. Br J Ophthalmol 2016;100(12):1686-91.

2. Newman A, Andrew N. Changes in Australian practice patterns for glaucoma management since the introduction of Minimally Invasive Glaucoma Surgery (MIGS). In Melbourne Ophthalmic Alumni Meeting; 2018 May 12; Melbourne, Australia.

3. Kerr NM, Wang J, Barton K. Minimally invasive glaucoma surgery as primary stand-alone surgery for glaucoma. Clin Exp Ophthalmol 2017;45(4):393-400. 4. Malvankar-Mehta MS, Iordanous Y, Chen YN, et al. iStent with Phacoemulsification versus Phacoemulsification Alone for Patients with Glaucoma and Cataract: A Meta-Analysis. PLoS One 2015;10(7):e0131770.

5. Malvankar-Mehta MS, Chen YN, Iordanous $Y$, et al. iStent as a Solo Procedure for Glaucoma Patients: A Systematic Review and Meta-Analysis. PLoS One 2015;10(5):e0128146.

6. The Advanced Glaucoma Intervention Study (AGIS): 7. The relationship between control of intraocular pressure and visual field deterioration. The AGIS Investigators. Am J Ophthalmol 2000;130(4):429-40.

7. Yook E, Vinod K, Panarelli JF. Complications of micro-invasive glaucoma surgery. Curr Opin Ophthalmol 2018;29(2):147-54.

8. Spiegel D, Wetzel W, Neuhann T, et al. Coexistent primary open-angle glaucoma and cataract: interim analysis of a trabecular micro-bypass stent and concurrent cataract surgery. Eur J Ophthalmol 2009;19(3):393-9.

9. Khouri AS, Megalla MM. Recurrent hyphema following iStent surgery managed by surgical removal. Can J Ophthalmol 2016;51(6):e163-e5.

10. Sandhu S, Arora S, Edwards MC. A case of delayed-onset recurrent hyphema after iStent surgery. Can J Ophthalmol 2016;51(6):e165-e7. 
11. Buchacra O, Duch S, Milla E, Stirbu O. One-year analysis of the iStent trabecular microbypass in secondary glaucoma. Clin Ophthalmol 2011;5:321-6. 12. Samuelson TW, Katz LJ, Wells JM, et al. Randomized evaluation of the trabecular micro-bypass stent with phacoemulsification in patients with glaucoma and cataract. Ophthalmology 2011;118(3):459-67. 


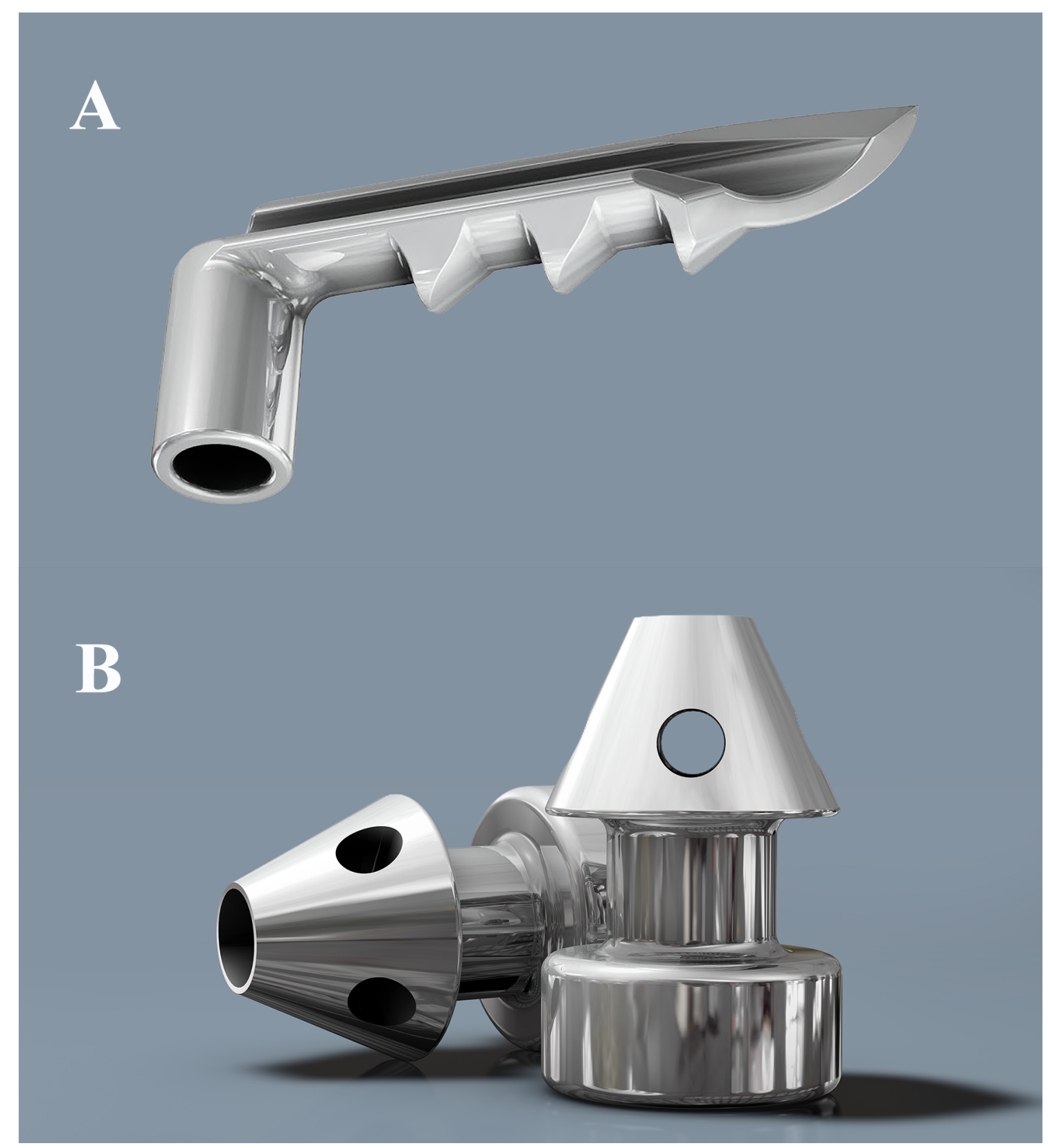

CE0_13526_figure_1.tif

This article is protected by copyright. All rights reserved. 


\section{University Library}

\section{- M M N E R VA A gateway to Melbourne's research publications}

Minerva Access is the Institutional Repository of The University of Melbourne

Author/s:

Hooshmand, J;Rothschild, P;Allen, P;Kerr, NM;Vote, BJ;Toh, T

Title:

Minimally invasive glaucoma surgery: Comparison of iStent with iStent inject in primary open angle glaucoma

Date:

2019-09-01

Citation:

Hooshmand, J., Rothschild, P., Allen, P., Kerr, N. M., Vote, B. J. \& Toh, T. (2019). Minimally invasive glaucoma surgery: Comparison of iStent with iStent inject in primary open angle glaucoma. CLINICAL AND EXPERIMENTAL OPHTHALMOLOGY, 47 (7), pp.898-903. https:// doi.org/10.1111/ceo.13526.

Persistent Link:

http://hdl.handle.net/11343/285870 